\title{
Balkanologie
}

Balkanologie Revue d'études pluridisciplinaires

Vol. IV, $n^{\circ} 1 \mid 2000$

Volume IV Numéro 1

\section{Le Temps et la question balkanique au début du $\mathrm{XX}^{\mathrm{e}}$ siècle}

Nadine Bonnefoi

\section{OpenEdition}

Journals

Édition électronique

URL : http://journals.openedition.org/balkanologie/783

DOI : 10.4000/balkanologie.783

ISSN : 1965-0582

\section{Éditeur}

Association française d'études sur les Balkans (Afebalk)

\section{Édition imprimée}

Date de publication : 1 septembre 2000

ISSN : 1279-7952

Référence électronique

Nadine Bonnefoi, «Le Temps et la question balkanique au début du xx siècle », Balkanologie [En ligne], Vol. IV, $n^{\circ} 1$ | 2000, mis en ligne le 03 juin 2008, consulté le 17 décembre 2020. URL : http:// journals.openedition.org/balkanologie/783; DOI : https://doi.org/10.4000/balkanologie.783

Ce document a été généré automatiquement le 17 décembre 2020.

(c) Tous droits réservés 


\title{
Le Temps et la question balkanique au début du $\mathrm{Xx}^{\mathrm{e}}$ siècle
}

\author{
Nadine Bonnefoi
}

1 Traditionnellement désignée comme le carrefour de l'Europe et de l'Asie, la péninsule des Balkans entre de nouveau, après la Grande Crise d'Orient de 1877-1878, dans la diplomatie internationale au cours des premières années du siècle et devient une question dominante des chancelleries européennes par l'enjeu qu'elle représente dans la formation des alliances.

2 Cette question, domine progressivement la vie politique et diplomatique de l'époque mais est l'objet d'un écho irrégulier dans la presse. En France, peu de journaux s'attachent aux problèmes des nationalités en Europe centrale et orientale. Préoccupés davantage par les questions économiques et coloniales qui secouent les relations franco-allemandes, ils ne distinguent pas encore le grand défi lancé à l'Europe par la question d'Orient. Plus visionnaire, Le Temps s'est sensibilisé au problème balkanique à travers l'opposition grandissante de la Serbie et de l'Autriche-Hongrie et ses conséquences sur l'équilibre général du continent. Mais le réveil des nationalités en Europe centrale et orientale reste plutôt mal compris. Les peuples des Balkans, considérés comme mineurs, restent les pions des grandes puissances qui ne peuvent tolérer des tensions risquant d'entraîner un conflit que tous veulent éviter. Si le journal admet l'existence de peuples opprimés cherchant à se rapprocher afin de s'émanciper de leurs tutelles, il n'envisage pas l'éclatement des grands Empires ni la création d'un État regroupant tous les Slaves du Sud. La diversité et la complexité du problème des peuples mêlé à celui des nationalités, tels qu'ils se présentent dans cette partie de l'Europe, sont encore mal perçues. Il est de bon ton, dans un pays comme la France qui se veut être le protecteur des droits de l'homme, de s'émouvoir pour des peuples qui luttent en son nom, si cela reste dans un cadre strictement pacifique et ne porte pas atteinte à l'ordre établi par les États dominants. «Il ne faut pas que la Serbie donne le spectacle de Byzance assiégée, si elle veut demeurer digne des sympathies sur lesquelles elle a le droit de compter. $»^{1}$ 
3 Le Temps a ainsi soutenu le choix européen du maintien de l'intégrité ottomane par crainte de voir surgir une multitude de mini-États risquant de déstabiliser la région et de l'entraîner dans la tourmente. Il a paré la France d'un droit supérieur que lui confère son rôle de grande puissance pour devenir la principale inspiratrice de la paix. « Dans la mesure où la presse peut contribuer à une œuvre internationale, notre concours a été acquis à celle-ci. Et non content d'en souhaiter le succès, nous y avons les premiers travaillé. $»^{2}$ Il a également demandé aux grandes puissances de jouer leur rôle de tutrices des petits peuples et d'abandonner ainsi leurs divisions pour adopter une politique d'union dans la recherche de la paix. Mais ces démarches sont restées sans effet car totalement subordonnées au maintien du statu quo et à l'intégrité ottomane. Les différentes nationalités doivent d'abord vivre en paix pour que l'Europe puisse considérer leurs justes revendications et proposer des réformes en leur faveur. " Il ne s'agit pas de livrer la Macédoine à la suprématie exclusive d'un élément quelconque. Ce qu'il faut, c'est, dans l'ordre et la paix, sous le contrôle de l'Europe, y assurer aux Slaves et aux Grecs, aux Chrétiens et aux musulmans les bienfaits du self-government, de la liberté et de la légalité. $»^{3}$

4 Le quotidien a toujours souhaité replacer le débat dans une perspective européenne. $\mathrm{Au}$ début du siècle, l'Europe des Nations domine, une Europe divisée, entrainée progressivement dans la formation de deux systèmes d'alliances aux ambitions conflictuelles. La volonté d'un éditorialiste comme Tardieu d'européaniser les problèmes balkaniques doit donc servir à un rapprochement des différents systèmes d'alliances autour d'une volonté commune d'apaiser les troubles dans une région que l'on pressent explosive.

Pour tous les esprits droits et non prévenus, tous les éléments qui se sont déroulés et qui se préparent en Macédoine démontrent avec une éclatante et irréfragable évidence que l'idée d'un contrôle européen s'impose, non seulement pour veiller à l'exécution loyale et sincère des réformes, mais pour prévenir par un déploiement opportun de forces un effroyable choc de barbaries ennemies. ${ }^{4}$

5 Le Temps n'a pas besoin de rappeler sa proximité géographique pour saisir le danger réel d'un conflit. Les ambitions expansionnistes des Empires dans cette partie de l'Europe, les sympathies et les haines des puissances envers les petits peuples sont alors beaucoup plus tranchées et ne sont freinées par aucune instance supérieure. Chaque État peut agir à sa guise, c'est à dire en fonction de sa force. « Pour être sûr de la paix, il n'y a qu'un seul moyen : c'est être prêt à la guerre. " " Quand les diplomates parlent, il faut considérer les armes pour savoir quel crédit on doit accorder à leurs propos. Mandataires des nations, leur autorité se mesure à la force de leurs mandats. $»^{6}$

6 La crise bosniaque de 1908 瞇 1909 est une alerte sérieuse. "On ne s'incline devant le droit que s'il a l'épée à la main. $»^{7}$ Le journal y découvre l'efficacité d'une diplomatie musclée reposant sur des forces armées substantielles. «En ce jour anniversaire, la seule conclusion qui s'impose, c'est que dans l'état présent de l'Europe, pour être sûr de son droit, il faut être sûr de sa force ; c'est que sans une armée, et la volonté de s'en servir au besoin, mieux vaut ne pas faire de la politique étrangère et se résigner à tout. $»^{8}$ Cette crise renforce également sa crainte d'une hégémonie austro-allemande sur la péninsule et lui ouvre les yeux sur le sort de la Serbie qui devient la principale barrière aux ambitions austro-allemandes et compte désormais parmi les grands défis européens.

Il y a, dans la crise orientale, deux sortes de forces à considérer: les forces

diplomatiques et les forces nationales. Il faut compter avec les secondes autant 
qu'avec les premières. Les Serbes dépassent de toutes parts le royaume de Serbie. Il est vain de nier leur existence et leur aspiration. Il est vain de donner pour artificielle une émotion qui est réelle et profonde, qu'il faut contenir et apaiser, mais qu'il est imprudent et inutile de méconnaître. ${ }^{9}$

7 Le grand quotidien aurait approuvé la pacification de la péninsule par les deux grands empires russe et austro-hongrois, mais leur incapacité à régler les problèmes ethniques en Macédoine et la mauvaise volonté de la Turquie lui font peu à peu préférer le règlement des troubles balkaniques par les États balkaniques eux-mêmes. «Elle (la politique européenne) se traîne dans la médiocrité des combinaisons dont la pauvreté étonne quiconque a lu l'histoire (...) on croit que c'est de la politique : c'est tout au plus de l'intrigue sans ampleur. Ce n'est pas la situation qui manque aux hommes. Ce sont les hommes qui manquent à la situation. $»^{10}$ Il utilise ainsi l'opposition croissante du peuple serbe à la politique autrichienne et son attachement naturel à la Russie pour se forger une attitude de plus en plus austrophobe et slavophile.

8 Tardieu plaide pour le rapprochement des États balkaniques dont l'indépendance effective lui paraît désormais indispensable pour établir un ordre durable dans la région et, par la suite, l'équilibre et la paix en Europe. «Il est mauvais que des États souverains soient en même temps des États faibles, et la faiblesse des pays balkaniques a été due pour beaucoup à leurs divisions. L'apaisement de ces querelles intestines serait, à nos yeux, un excellent progrès. " ${ }^{11}$ Mais ce rapprochement doit rester strictement $\mathrm{du}$ domaine économique et l'éditorialiste répète fréquemment son attachement au statu quo dont la puissance de l'armée turque fait figure de dernier défenseur.

9 L'engagement des hostilités entre Turcs et Alliés balkaniques et la victoire inattendue de ces derniers en 1912 placent l'Europe tout entière devant un fait accompli dont le journal s'accommode très rapidement. "L'Europe, par son attitude, a convaincu les États balkaniques qu'on n'obtient rien d'elle par la douceur. "Mieux vaut violence", voilà la maxime du jour. Et ce sont les puissances qui ont donné cours à cette maxime. $»^{12} \mathrm{Il}$ y voit un nouveau moyen pour les grandes puissances de s'entendre sur la reconnaissance des faits accomplis en s'assurant du caractère résolument pacifique des États ainsi constitués. Il s'agit, en quelque sorte de légitimer une agression en échange d'une paix globale très hypothétique. Le déclenchement du deuxième conflit entre alliés de la veille provoque un brusque repli de l'éditorialiste qui décrit la péninsule comme le théâtre d'une folie meurtrière collective dont la seule sauvegarde pour l'Europe réside dans l'isolement complet des belligérants. «(...) Les États balkaniques, par leur façon de procéder, ont fait le vide autour d'eux. S'il leur plat de s'entr'égorger et de se diminuer eux-mêmes, ils détourneront en quelques sens que ce soit, les grandes puissances de les soutenir. $»^{13}$ «En cette guerre fratricide, il ne peut y avoir que des vaincus. $»^{14}$

10 Tardieu se rassure pourtant : aucun conflit brutal n'a surgi entre la Triple-Alliance et la Triple-Entente lors des deux conflits balkaniques. Il se rallie alors à l'idée d'un rapprochement de la France vers les puissances qu'il croit sortir vainqueurs du conflit et qui deviendraient des clientèles économiques et diplomatiques précieuses. Cette recherche de nouvelles clientèles marque la volonté des grandes puissances de développer leur propre crédit et s'accompagne inévitablement d'un resserrement autour de leurs alliances et donc de leur durcissement. Elle ouvre de nouveau la voie aux réactions individuelles des grands empires dont l'intervention ne peut plus être 
déterminée par un mandat international mais par la seule volonté du plus fort d'imposer ses vues au plus faible.

11 Inconscient des dangers d'une telle course aux alliances, Le Temps se fait l'apôtre d'un rapprochement de la France et des États balkaniques nouvellement émancipés comme une manifestation logique de la ligne générale de la politique extérieure de la France dirigée vers l'alliance avec la Russie. "(...) Tout révèle à la Serbie que l'évolution présente de la France fournit désormais au slavisme le point d'appui voulu, pour le fortifier dans sa lutte contre les puissances allemandes ou, le cas échéant, pour faciliter avec elles une plus favorable entente (...) $»^{15}$ Cette précieuse union oppose la seule barrière efficace à la poussée austro-allemande en Europe du sud-est et garantit de ce fait l'équilibre général du continent. Se rapprocher des États chrétiens vainqueurs des Turcs, c'est donc à la fois soutenir leurs positions nouvelles face aux ambitions tripliciennes tout en renforçant les liens avec la Russie, protectrice naturelle des Slaves.

Le renforcement militaire est la dernière étape pour rendre la France inattaquable. Le Temps a tiré une leçon des crises successives : l'importance de la force des armes sur la force des lois. «L'état de nos forces militaires est l'élément capital de notre police extérieure. C'est l'encaisse militaire de chaque peuple qui mesure son crédit diplomatique. ${ }^{16} \mathrm{Il}$ défend donc énergiquement l'idée d'un renforcement militaire et aide de tout son pouvoir l'adoption de la loi des 3 ans. Il s'associe ainsi à l'assentiment patriotique d'une grande partie de la nation et couvre les protestations des socialistes et de quelques intellectuels.

13 La course aux armements et aux effectifs s'accélère dans chaque camp, entretenant la méfiance et rendant permanente la tension internationale. Aveuglé par l'idée d'équilibre et de paix armée, Le Temps n'a pas réalisé les conséquences logiques de l'opposition des alliances et des intérêts sur l'évolution d'une Europe fragilisée par les passions individuelles et les idées nationales. La valeur de l'équilibre des forces doit avant tout calmer l'arrogance des puissances et l'exaspération des populations.

14 Le conflit qui éclate en été 1914 est totalement imputé à l'Autriche-Hongrie et aux volontés hégémoniques austro-allemandes. «C'est parce que l'Allemagne et l'Autriche voulaient sceller définitivement leur hégémonie, en Orient d'abord et ailleurs ensuite, que la Russie a été touchée, et avec elle tout le corps européen. (...) $\rrbracket^{17}$ Promptement convaincu de l'inéluctabilité d'une conflagration que les Alliés, ultimes défenseurs du droit et de la justice, acceptent courageusement, Le Temps garde une confiance absolue dans la victoire finale.

15 La Triple-Entente, ouverte à toutes les bonnes volontés, devient le champion de la lutte des peuples pour la liberté et l'indépendance. « (...) Nous nous bornerons à répéter très nettement que les alliés resteront vainqueurs (...) et que ceux qui les auront aidés ou combattus seront traités équitablement, en tenant compte des droits des nationalités, suivant leurs mérites et leurs gestes. ${ }^{18}$ Les empires centraux sont désignés, quant à eux, comme des barbares dont l'incurable volonté dominatrice doit être à jamais anéantie pour aboutir enfin à l'Europe des droits et des libertés.

16 L'inévitable effondrement de l'Autriche-Hongrie s'annonce progressivement et le journal ne cesse d'insister sur les signes d'affaiblissement de l'Empire dont l'opposition systématique à l'affirmation nationale que revendique la majorité des peuples qui le composent. "L'empire austro-hongrois n'a désormais plus en perspective que ses démembrements. $»^{19}$ Dans le cadre de ce qui peut s'appeler la Slavie du Sud, il considère généralement l'unité totale de culture et de race de ces peuples et légitime dorénavant 
leur volonté d'union sous l'égide de la Serbie. "Les Jougo-Slaves ne tarderont pas à célébrer leur réunion à la commune patrie. La Serbie aura réussi ainsi à gagner par ses propres forces ce que d'autres paraissent attendre de la faveur des événements. $»^{20} \mathrm{Il}$ admet les plaintes des Sud-Slaves de l'Empire contre un pouvoir qu'ils jugent oppressif mais il ne leur tient compte d'aucune volonté particulière d'indépendance et leur prête le seul désir d'une union avec leurs frères de Serbie. " Nous sommes tous, nous voulons tous être citoyens de la même grande Serbie. (...) N'en doutez pas! Nous sommes et nous voulons ne faire qu'un seul et même peuple: catholiques, orthodoxes ou musulmans, qu'importe. Nous sommes et nous resterons une race, une nation. $»^{21}$ Cette dernière a un rôle évident de rassembleur et de dirigeant des peuples slaves du Sud. Elle peut se limiter à la volonté d'une grande Serbie réunissant tous les peuples de race serbe ou envisager la formation d'un État associant tous les peuples slaves du sud de l'Autriche-Hongrie.

$1^{\circ}$ En ces jours historiques de sacrifices et d'espérance dans le droit et la liberté, nous affirmons d'abord notre pleine et indivisible unité nationale serbo-croatoslovène et cela, non seulement pour l'amélioration de l'avenir, mais pour la cause des vérités ethnographiques qui doit se réaliser politiquement comme elle l'est moralement.

$2^{\circ}$ En conséquence, nous déclarons sans aucune réserve que nous protesterons tant que nos pays purement sud-slaves seront sacrifiés, déchirés, sur tout le littoral où vivent Serbes-Croates-Slovènes.

$3^{\circ}$ Nous prions toutes les puissances qui luttent aujourd'hui pour le principe des nationalités et la justice de sauvegarder notre race indivisible de ce déchirement qui la frustre de ses territoires et de rendre possible à la Serbie la mission civilisatrice qui sera la condition d'une longue paix en Europe. ${ }^{22}$

17 Le journal soutient nettement son rôle directeur dans un avenir volontairement enveloppé d'un flou qui le maintient toujours ouvert. Un État indépendant et fort de près de 10 millions d'habitants sous la dynastie des Karageorgévitch formerait un rempart définitif aux ambitions austro-allemandes et serait un interlocuteur privilégié de la France qui a su maintenir de bonnes relations avec le pouvoir serbe. Le Temps a ainsi largement laissé entendre que les dures raisons de la politique internationale coïncident parfaitement, dans ce cas, avec les enjeux et les raisons idéales de la nationalité.

La défection des Russes et la crainte d'un abandon total de l'Orient aux puissances ennemies renforcent l'attachement déjà manifeste du journal envers la Serbie. «Qu'elle soit provisoire ou définitive, la faiblesse de la Russie ne peut que rendre plus précieuses les positions occupées par les alliés en Macédoine et en Moldavie. Si la Russie doit se ressaisir, ces positions sont des points d'appui indispensables pour la tâche que les alliés auraient à accomplir en Orient. Si la Russie est perdue sans espoir, ce sont des gages inestimables en vue du règlement final. $»^{23}$ Grâce à leur étroite cohésion, les Serbes ont duré, se sont émancipés et ont acquis, dans la guerre, un titre définitif. La restauration de leur indépendance est un des principaux buts de guerre auquel s'ajoute la liberté des peuples à disposer d'eux-mêmes qui doit permettre aux Sud Slaves de la Double Monarchie de décider de leur avenir.

Serbes, Croates et Slovènes veulent vivre ensemble, sous le même gouvernement et en complète indépendance. Ce qu'ils réclament, c'est leur droit à l'existence au même titre que toutes les autres nations de l'Europe. Nulle autre solution que celleci ne sera viable. Et si l'on comprend bien les raisons intéressées qu'a l'Autriche de s'y opposer, on ne voit pas au nom de quels principes les alliés refuseraient d'écouter la voix de ses peuples. ${ }^{24}$ 
Mais Le Temps leur laisse-t-il vraiment le choix lorsqu'il leur démontre le peu de crédit que leur porteront les Alliés s'ils ne témoignent d'aucune manifestation concrète de leur lutte contre le pouvoir austro-hongrois et s'ils ne s'unissent pas volontairement à leurs frères de Serbie? Seuls, ces derniers pourront les représenter utilement aux négociations de paix et obtenir leur indépendance totale. «Disons aux nationalités slaves et latines de l'Autriche-Hongrie : "Votre cause est en jeu, comme la nôtre, dans la bataille du front occidental. Luttez, par tous les moyens pour disloquer la puissance germanique. Vos libertés futures se mesureront aux services que vous rendez à présent !". ${ }^{25}$ Doivent-ils encore hésiter devant des détails qui ne font qu'entacher une unité reconnue de tous?

Le Temps ne cesse de répéter aux peuples slaves du sud de l'Autriche-Hongrie qu'ils ont tout à gagner à s'unir dans un État souverain et indépendant dont les autorités serbes assureraient les valeurs démocratiques.

21 Cette union, envisagée comme l'aboutissement d'un choix libre et délibéré renforcerait son poids de future alliée de la France et mettrait définitivement hors d'état de nuire les empires centraux. Le Temps occulte donc totalement les menaces extérieures (Italie) dans la décision croate et slovène d'une union avec la Serbie. Il préfère considérer l'élan des cœurs et la victoire morale de la liberté et de l'indépendance des peuples pour légitimer définitivement la création d'États constitués, indépendants, fondamentalement anti austro-allemands et naturellement attachés aux puissances de l'Entente. « Mais il faut le dire et le redire : puisse cette unité yougoslave s'incarner au plus tôt dans un groupe d'hommes qui auront qualité pour parler au nom de tous les Serbes, de tous les Croates et de tous les Slovènes. Le "peuple aux trois noms" doit s'exprimer d'une seule voix. $»^{26}$

Résolument opportuniste, Le Temps a suivi l'évolution des questions nationales en Europe centrale et orientale en les replaçant immédiatement dans le contexte général du Continent. Il a ainsi accompagné de son pouvoir la politique générale de la France et l'a poussé selon ses propres convictions dans une pseudo psychose d'un conflit généralisé contre lequel tout devait être mis en œuvre diplomatiquement et militairement, tout en légitimant ces mesures comme la meilleure garantie de paix. Son attachement à la question serbe et Sud-slave tient donc de l'utilisation d'un problème frappant de plein fouet des empires appartenant à un bloc d'alliances opposé et de son intérêt pour la France qui cherche à s'imposer de nouveau dans le concert européen. Il a soutenu l'important effort de redressement militaire et diplomatique de la France en légitimant cette volonté par la description de plus en plus alarmiste des troubles répétés et incontrôlables dont l'incapacité des grandes puissances à les résoudre ne leur laisse plus d'autre choix que de s'en protéger elles-mêmes.

23 Il est néanmoins l'un des rares à s'être sensibilisé aux problèmes nationaux et à les avoir exposés à une opinion publique ignorante et préoccupée par d'autres questions. Son analyse n'a pourtant jamais été totalement libre et dénuée d'arrières pensées. Elle s'est toujours trouvée bloquée par des considérations supérieures diverses et équivoques.

24 Le Temps a-t-il eu réellement peur d'un conflit généralisé dont il présageait la ruine pour l'Europe? A-t-il fidèlement adhéré ou contribué à ces replis diplomatiques et militaires en se persuadant de leurs effets décisifs sur le maintien de la paix générale? S'est-il laissé aveugler à l'instar des grandes puissances qui, croyant se protéger d'un 
conflit prévisible, se sont elles-mêmes lancées dans l'aventure ? Conscient peu à peu d'un péril grandissant, aurait-il été fasciné par lui ?

Il est certes difficile d'évaluer dans quelle mesure il y eut une manipulation exercée mais aussi subie par le journal. Celle-ci semble avoir néanmoins existé et s'être développée tout au long de la période. Les peuples slaves des Balkans ont compris, au début du siècle, l'importance de l'opinion publique européenne et ont découvert progressivement la puissance de l'information journalistique qu'ils ont utilisé pour intensifier la propagande slavophile.

Prétextant participer à l'édification d'un équilibre européen, Le Temps a largement été influencé par des gouvernements étrangers desquels il a touché des sommes importantes. Les journalistes corrompus devaient écrire des articles très favorables mais bien souvent ils se contentaient de reproduire des dépêches dictées directement par ceux qui en payaient la parution. André Tardieu, chef des services de politique étrangère pendant près de dix ans et Edgard Roels, fondateur de l'agence des Balkans, se faisaient ainsi payer leurs bonnes grâces en diffusant des informations touchant près de $10 \%$ des nouvelles publiées en France à propos des Balkans. Cette corruption, très répandue avant 1914 et qui deviendra endémique après la guerre servait dans un premier temps les ambitions économiques et financières des États payeurs. Une presse française favorable devait par exemple permettre à la Russie d'obtenir des émissions d'emprunts indispensables à son redressement intérieur et notamment un redressement militaire lui permettant de jouer pleinement son rôle au sein de l'alliance.

27 L'influence politique et diplomatique de cette propagande est certaine. Elle l'est d'autant plus dans le cas du Temps car les colonnes de politique étrangère étaient toutes l'œuvre d'une seule main. André Tardieu joua ce rôle de 1905 à 1914. Son analyse, d'après les dépêches de l'Agence des Balkans n'était associée à aucune opinion extérieure si ce n'est celle des hommes politiques impliqués dans cette propagande. La toute puissance de l'éditorialiste devenait un instrument idéal de manipulation des peuples et des gouvernements. La publication par les Soviets, en 1917, de correspondances attestant de l'étendue de la corruption de la presse française, est édifiante. Ancien économiste russe, A. Raffalovitch était chargé de cette délicate entreprise. Une bonne partie de la presse française était touchée et Le Temps, considéré comme l'un des journaux les plus importants de Paris, était copieusement soudoyé. Des lettres d'Iswolski, alors ambassadeur de Russie en France, confirment ainsi, dans les dernières années précédant la guerre, la parfaite docilité de Tardieu aux consignes du cabinet russe. Des sommes considérables ont été allouées aux journalistes prêts à mettre leur plume à la disposition des plus offrants. La Russie a su se montrer généreuse. Elle a su également choisir ses journalistes et se ménager les bonnes grâces de celui qui deviendra plus tard l'un des négociateurs de la paix.

Le Temps a également rendu certains services à son gouvernement, par patriotisme plus que par intérêt, en filtrant des informations, en retardant leur diffusion ou en orientant partiellement leurs commentaires. Ce patriotisme se manifestait surtout en période de crise, par l'acceptation d'une sorte de tutelle temporaire des autorités. La censure, détestée en temps de paix, s'est imposée pendant la guerre, Le Temps acceptant l'obéissance à l'autorité comme une des premières manifestations du civisme. 
Il a malgré tout critiqué à plusieurs reprises le pouvoir et la censure, mais celle-ci a été très clémente. Rares sont les "blancs". Le Temps s'est montré généralement prudent en matière de politique étrangère et n'a que très peu subit de coupures.

Ses querelles concernaient surtout la censure politique utilisée par les gouvernements pour réduire le rôle de la presse dans le débat de politique intérieure, atténuer l'expression des oppositions de partis et s'approprier les faveurs de l'opinion publique.

31 Les censures militaires et diplomatiques ont rencontré, au contraire, peu de résistance. Le Temps a généralement suivi fidèlement les consignes des autorités et a reproduit des nouvelles et des commentaires déjà contrôlés, devenant un docile messager d'une propagande et d'une manipulation officielle. Ainsi, rien n'a filtré des nombreuses intrigues et négociations qui ont précédé l'entrée en guerre de l'Italie. Ceci est également flagrant pour les premières nouvelles sur l'explosion révolutionnaire en Russie qui furent retardées et épurées pour éviter au peuple français le choc trop brutal de l'abandon de ses anciens alliés. La lecture du journal montre combien le traitement de ces deux événements fut imparfait et délibérément orienté.

Ses rapports avec la censure militaire n'ont connu que quelques sujets de discorde. Fervent défenseur du front oriental, il dut subir l'opposition de responsables militaires et politiques attachés au seul front occidental. Plusieurs dépêches sur les opérations de l'armée d'Orient furent ainsi censurées, empêchant l'opinion publique d'être informée et donc réellement sensibilisée à ce sujet. La censure militaire, au-delà de la simple interdiction de publier des informations précises sur les opérations en cours, servait également les intrigues de l'état-major, la manipulation consistant alors à écarter volontairement de l'actualité des journaux, des grands noms de chefs militaires comme Castelnau ou Sarrail.

Le Temps a toujours connu, en temps de paix comme en temps de guerre, certains désaccords avec les pouvoirs gouvernementaux. Durant le conflit mondial, la censure a $\mathrm{du}$ intervenir pour calmer ses ardeurs opportunistes mais son patriotisme et son loyalisme se sont imposés.

Reproduisait-il simplement les informations des milieux officiels, seuls responsables de cette manipulation ou bien s'est-il montré volontairement conciliant envers ces mêmes autorités pour participer, avec elles, au contrôle de l'opinion publique, contribuer à "l'anesthésier" et rendre possible les opérations de propagande?

En règle générale et sur l'ensemble de la période, Le Temps a souhaité influencer les hommes politiques plutôt que les opinions publiques. Il a toujours agi selon les circonstances en formulant des revendications précises et en invitant fermement les dirigeants français et étrangers à se laisser guider par ses propositions. Son action sur l'opinion publique a été beaucoup plus paralysante. Concernant l'épineuse question du réveil des nationalités en Europe centrale et orientale, il a trop souvent obscurci la compréhension des oppositions ethniques. Il a successivement diabolisé les différentes nationalités pour maintenir le public français dans une incompréhension totale de la situation, bloquant toute réaction et donc toute influence sur le pouvoir politique. Dangereusement versatile, il est resté prisonnier d'une émotivité qui l'a conduit, dans un premier temps, à une grande indulgence envers ces peuples opprimés cherchant la liberté : «l'Europe, par son attitude, a convaincu les États balkaniques qu'on n'obtient rien d'elle par la douceur. "Mieux vaut violence", voilà la maxime du jour. Et ce sont les puissances qui ont donné cours à cette maxime ${ }^{27}$, puis les a violemment condamnés 
pour s'être engagés dans une guerre fratricide risquant de troubler l'ordre européen : " ...les États balkaniques, par leur façon de procéder, ont fait le vide autour d'eux. S'il leur plaît de s'entr'égorger et de se diminuer eux-mêmes, ils détourneront en quelque sens que se soit, les grandes puissances de les soutenir... Les grandes puissances, croyons-nous, sont dès maintenant d'accord pour tracer autour des Balkans un cordon sanitaire et se protéger contre les conséquences d'un accès de démence collective, qui ne peut s'apaiser que dans l'isolement ${ }^{28}$, pour enfin se retrancher derrière l'argument, témoin de son échec : tout est trop complexe, tout le monde a tort, "il serait puéril et fastidieux de les départager. Tous, chacun à son heure, se sont mis dans leur tort ${ }^{29}$.

L'analyse équitable des diverses nationalités disparaissait bien souvent pour ne tenir compte que des rivalités politiques et diplomatiques de l'Europe et se limiter à la recherche de sympathies individuelles capables de rehausser le prestige de la France et d'assurer au bloc de la Triple-Entente la meilleure sécurité. Le choix de la Serbie, la plus hostile aux puissances austro-germano-turques et dont la communauté de race l'attache nécessairement aux Russes, s'est imposé au gré des événements politiques, diplomatiques et militaires locaux mais aussi européens. Ce choix n'a cessé de s'affirmer, malgré certains achoppements lorsque Le Temps estimait, avec beaucoup de condescendance, qu'elle allait trop loin et devait se contenter de ses conseils de sagesse.

Pendant la guerre, il a véritablement mythifié le peuple serbe et a contribué de façon évidente à imposer son charisme et son rôle naturel d'unificateur et de rassembleur des peuples. Parallèlement et suivant la même évolution, son jugement envers l'AutricheHongrie n'a cessé de se durcir pour aboutir à l'image, reprise de longues années durant par l'historiographie contemporaine de "prison des peuples". L'Empire, voué à disparaître, n'était plus qu'une combinaison politique et étatique malsaine et dangereuse dès lors qu'elle devenait l'outil de l'impérialisme allemand

Le successeur de Tardieu pendant les quatre années du conflit, Roland de Marès, s'est à son tour fait l'apôtre du sens divin de la lutte des Serbes contre la barbarie et leur a promis une destinée exceptionnelle. Ce peuple, dont l'existence au sein d'un État considérablement élargi aux dépens des anciens empires, redonne à la France, déstabilisée par la nouvelle indépendance politique et diplomatique de la Russie, une place prépondérante dans la future Europe.

39 En faisant très tôt le choix de la Serbie et en adoptant définitivement les revendications nationales serbes, Le Temps s'est laissé entraîner dans une opposition systématique à l'Autriche-Hongrie et a opté définitivement pour l'éclatement de l'Europe des Nations au profit d'une Europe des Nationalités qui devait sonner le glas des ambitions expansionnistes et instituer une paix internationale basée sur le droit des peuples à disposer d'eux-mêmes.

40 Mais si le démantèlement de l'Autriche-Hongrie s'est fait sur «le principe des nationalités ", les États successeurs sont à nouveau des pays multinationaux et la Serbie, État cosmopolite, regroupant des populations de traditions religieuses, politiques et culturelles diverses, en est un parfait exemple.L'idée yougoslave, c'est-àdire la réunion des Slaves du Sud, a triomphé mais qu'en est-il de l'esprit démocratique apparu en 1903 ?

41 Le royaume des Serbes, Croates et Slovènes est né dans l'enthousiasme mais aussi dans la hâte et dans une certaine confusion. Les équivoques pesant sur sa naissance n'ont d'ailleurs pas tardé à éclater au grand jour.Des dépêches sont ainsi publiées au 
lendemain de la guerre dans lesquelles les dirigeants monténégrins dénoncent l'annexion forcée à la Serbie. Ils sont assimilés à des Serbes, ce que beaucoup d'entre eux n'admettent pas.

Un conflit va également surgir lorsque sera élaborée la future constitution du royaume entre les partisans du centralisme, c'est-à-dire les vieux radicaux panserbes et ceux du fédéralisme qui réunissent les anciens membres de l'empire austro-hongrois, Slovènes, Croates et Serbes de Hongrie.

43 Ces dépêches publiées après la guerre devraient faire l'objet d'une recherche et d'une analyse plus approfondie car elles créent un paradoxe troublant avec les témoignages antérieurs d'unité totale des Yougoslaves et de la grande valeur morale de la nation serbe.

Il reste cette volonté de sécurité et de prestige international que Le Temps a toujours voulu servir et qui se solde, au lendemain du conflit par l'élimination de l'Europe orientale des Russes, Allemands et Autrichiens et par la nouvelle place des États balkaniques sous la protection de la France et l'Angleterre.

\section{NOTES}

1. Bulletin de l'étranger, «Le nouveau ministre serbe », Le Temps, 19/01/09, p.1.

2. Bulletin de l'étranger, « La France et l'alliance russe », Le Temps, 02/02/05, p.1.

3. Bulletin de l'étranger, « Macédoine », Le Temps, 31/12/03, p.1.

4. Bulletin de l'étranger, «Balkans », Le Temps, 04/04/03,p.1.

5. Bulletin de l'étranger, «Fin d'année », Le Temps, 01/01/07, p.1.

6. Bulletin de l'étranger, «De Brindisi à Vienne », Le Temps,15/05/09, p.1.

7. Bulletin de l'étranger, « Fin de crise », Le Temps, 30/03/09, p.1.

8. Bulletin de l'étranger, « Anniversaire », Le Temps, 07/10/09, p.1.

9. Bulletin de l'étranger, «Le conflit austro-serbe », Le Temps, 06/01/09.

10. Bulletin de l'étranger, «Fin d'année », Le Temps, 02/01/09.

11. Bulletin de l'étranger, «Le voyage du roi de Bulgarie », Le Temps, 29/10/10.

12. Bulletin de l'étranger, "Le rôle des puissances ", Le Temps, 03/10/12.

13. Bulletin de l'étranger, «La démence balkanique », Le Temps, 04/07/13.

14. Bulletin de l'étranger, «La douloureuse », Le Temps, 05/07/13.

15. Documents diplomatiques français, $M$. Descos, Ministre de France à Belgrade à M. Jonnart, Ministre des Affaires étrangères. Dépêche n53. Belgrade, 23/02/13.

16. Bulletin de l'étranger, «Les droits et les devoirs ", Le Temps, 14/06/14.

17. Bulletin de l'étranger, «Au sud du Danube », Le Temps, 12/09/14, p.1.

18. Bulletin de l'étranger, «Les demandes bulgares », Le Temps, 28/11/14, p.1.

19. Bulletin de l'étranger, « Le crépuscule austro-hongrois », Le Temps, 16/04/15, p.1.

20. «La Guerre. La situation diplomatique », Le Temps, 12/09/14, p.1.

21. «La Croatie et les Serbes », Le Temps, 29/09/14, p.2.

22. Nouvelles de l'étranger, « Une assemblée yougo-slave en Serbie », Le Temps, 15/05/15, p.2.

23. Nouvelles de l'étranger, « Macédoine et Moldavie », Le Temps, 28/12/17, p.2.

24. «Sur le front de Macédoine. L'arrivée des troupes jougo-slaves », Le Temps, 16/02/18, p.2. 
25. Bulletin de l'étranger, «Les alliés de l'Autriche-Hongrie », Le Temps, 03/06/18, p.1.

26. Bulletin de l'étranger, «La délivrance de Belgrade », Le Temps, 04/11/18, p.1.

27. Bulletin de l'étranger, «Le rôle des puissances », Le Temps, 03/10/12, p.1.

28. Bulletin de l'étranger, «La démence balkanique », Le Temps, 04/07/13, p.1.

29. Ibid.

\section{AUTEUR}

\section{NADINE BONNEFOI}

Nadine Bonnefoi est historienne. 